Portland State University

PDXScholar

Electrical and Computer Engineering Faculty

Publications and Presentations

$1-1-1974$

\title{
Air Breakdown in a Radial-Mode Focusing Element
}

Lee W. Casperson

Portland State University

Mohammad Shabbir Shekhani

University of California, Los Angeles

Follow this and additional works at: https://pdxscholar.library.pdx.edu/ece_fac

Part of the Electrical and Computer Engineering Commons

Let us know how access to this document benefits you.

\section{Citation Details}

Lee W. Casperson and Mohammad S. Shekhani, "Air Breakdown in a Radial-Mode Focusing Element," Appl. Opt. 13, 104-108 (1974).

This Article is brought to you for free and open access. It has been accepted for inclusion in Electrical and Computer Engineering Faculty Publications and Presentations by an authorized administrator of PDXScholar. Please contact us if we can make this document more accessible: pdxscholar@pdx.edu. 


\title{
Air Breakdown in a Radial-Mode Focusing Element
}

\author{
Lee W. Casperson and Mohammad S. Shekhani
}

\begin{abstract}
A new radial focusing device is described that condenses an incident laser beam to an extremely intense and uniformly illuminated focal spot. The focal region is useful for many applications. When used with a $10.6-\mu \mathrm{m} \mathrm{CO} \mathrm{CO}_{2}$ TEA. laser source, a disk-shaped air-breakdown spark results, and the properties of this spark have been investigated.
\end{abstract}

\section{Introduction}

An important application of focusing devices involving lenses or mirrors is to collect the energy of an incident beam and bring that energy to a sharply defined focus. In gas breakdown investigations, for example, the beam from a high powered laser is focused in a gas and the resulting fields are sufficient to ionize the gas and cause a breakdown spark. ${ }^{1}$ Such studies are necessary to determine the intensity limitations for beam propagation in the atmosphere and within the lasers themselves. The resulting breakdown sparks are also useful for fundamental plasma studies, and the recent $\mathrm{cw}$ sparks promise to be especially convenient. ${ }^{2,3}$ Efficient focusing and energy coupling are also required for optically pumped systems such as cw dye lasers and for laser zone refining of high purity materials. ${ }^{4}$ Perhaps the most stringent focusing requirements are those of the proposed laser fusion energy sources. The focusing elements of laser fusion devices must be capable of bringing extremely high powered beams to a small symmetrically illuminated spot. This focusing ability is essential for pellet compression, which is a basic feature of current laser fusion proposals. ${ }^{5}$

The purpose of this work is to describe a new type of radial focusing device involving a $360^{\circ}$ wraparound mirror. This kind of focusing element is straightforward to construct and has much better energy concentration and symmetry properties than a conventional spherical mirror. Also, the focal region is readily accessible for experimental applications.

In Sec. II are derived the energy focusing properties of three basic mirror configurations - the ideal deep paraboloid, the radial-mode element, and the simple spherical mirror. These latter two devices

\footnotetext{
The authors are with the School of Engineering and Applied Science, University of California, Los Angeles, California 90024.

Received 27 April 1973
}

can in some respects be regarded as segments of a paraboloidal mirror. In Sec. III is a description of the first experiments involving a radial-mode mirror. The mirror condenses in air the beam from a conventional 100-MW $\mathrm{CO}_{2}$ TEA laser, yielding a thin diskshaped breakdown spark $1.5 \mathrm{~cm}$ in diameter. Timeresolved measurements show that the spark expands rapidly as a laser-driven shock followed by a fairly uniform plasma glow lasting for about $5 \mu \mathrm{sec}$. This behavior is analogous to the upstream propagation of the breakdown spark near the focus of an ordinary spherical mirror.

\section{Theory}

In this section the properties of various basic types of focusing mirrors are briefly derived and compared, and the new radial focusing device is introduced. Important considerations are the energy focusing ability, ease of fabrication, and uniformity and accessibility of the focal region. Figure 1 is a schematic drawing of a general deep paraboloidal mirror. In terms of energy focusing a mirror of this type represents the ideal configuration. An incident plane wave is efficiently coupled into the well-known low order spherical resonator modes providing a maximum energy density at the focus. In particular, a radially polarized incident wave of appropriate amplitude distribution can couple its energy into the lowest order azimuthally symmetric TM spherical resonator mode. The field components of this mode may be written in complex form as $^{6}$

$$
\begin{gathered}
H_{\phi}=(C \sin \theta / k R)[(\sin k R / k R)-\cos k R], \\
E_{R}=-\left[2 i(\mu / \epsilon)^{1 / 2} C \cos \theta / k^{2} R^{2}\right][(\sin k R / k R)-\cos k R],
\end{gathered}
$$

$$
\begin{aligned}
E_{\theta}=\left[i(\mu / \epsilon)^{1 / 2} C \sin \theta / k^{2} R^{2}\right] & {\left[\left(k^{2} R^{2}-1\right)\right.} \\
\times & \times(\sin k R / k R)+\cos k R],
\end{aligned}
$$

where $C$ is a normalization constant.

A versatile figure of merit for focusing elements is the energy density at the focus per unit of incident 


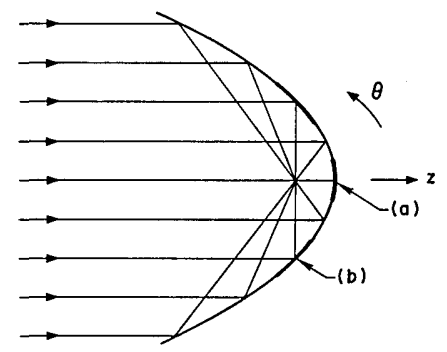

Fig. 1. Cross section through a paraboloidal focusing mirror. On the figure are marked $(a)$, a spherical mirror segment and (b), a radial focusing mirror segment.

power. The energy density in an electromagnetic field is given by

$$
u=(\epsilon \bar{E} \cdot \bar{E} *+\mu \bar{H} \cdot \bar{H} *) / 4 .
$$

Combining Eqs. (1)-(4) we find that the energy density of the lowest order mode is

$$
\begin{gathered}
u(R, \theta)=\left(\mu C^{2} \cos ^{2} \theta / k^{4} R^{4}\right)[(\sin k R / k R)-\cos k R]^{2} \\
+\left(\mu C^{2} \sin ^{2} \theta / 4 k^{2} R^{2}\right) \\
\times\left\{\left(1 / k^{2} R^{2}\right)\left[\left(k^{2} R^{2}-1\right)(\sin k R / k R)+\cos k R\right]^{2}\right. \\
\left.+[(\sin k R / k R)-\cos k R]^{2}\right\} .
\end{gathered}
$$

The energy density at the focus can be found by considering the limit $k R \rightarrow 0$ and the result is

$$
u(0)=\left(\mu C^{2} \cos ^{2} \theta / 9\right)+\left(\mu C^{2} \sin ^{2} \theta / 9\right)=\mu C^{2} / 9 .
$$

In the opposite limit of large distances $(k R \gg 1) \mathrm{Eq}$. (5) reduces to

$$
u(R, \theta)=\mu C^{2} \sin ^{2} \theta / 4 k^{2} R^{2} .
$$

Using Eqs. (6) and (7), the intensity of the inward traveling wave component $I(R, \theta)=c u(R, \theta) / 2$ can be related to the energy density at the focus by

$$
I(R, \theta)=\left(9 c \sin ^{2} \theta / 8 k^{2} R^{2}\right) u(0) .
$$

The total input power is obtained by integrating the intensity at large distances from Eq. (8) over the surface of a sphere of radius $R$ according to

$$
P=\int_{0}^{2 \pi} \int_{0}^{\pi} I(R, \theta) R^{2} \sin \theta d \theta d \phi=\left(3 c \lambda^{2} / 4 \pi\right) u(0)
$$

or

$$
u(0)=4 \pi P / 3 c \lambda^{2} .
$$

This is the best focusing possible for a beam of wavelength $\lambda$.

Two regions of the paraboloidal mirror shown in Fig. 1 are of special interest, and they are marked by the letters $(a)$ and $(b)$. These areas by themselves represent important focusing elements. The end cap of the paraboloidal mirror is the familar simple spherical mirror. The properties of spherical mirrors are well known, and they are indicated here for comparison purposes. To calculate the focusing ability, assume that the incident beam is a plane Gaussian wave having an $e^{-2}$ intensity spot size $w$ equal to the radius of the mirror. The mirror spot size is related to the spot size at the beam waist $w_{0}$ a distance $z$ from the mirror by the familiar expression ${ }^{7}$

$$
w=w_{0}\left[1+\left(z \lambda / \pi w_{0}^{2}\right)^{2}\right]^{1 / 2} .
$$

For a strongly focusing mirror $\left(w \gg w_{0}\right)$ this reduces to $w=z \lambda /\left(\pi w_{0}\right)$. In terms of the $f$ number $f=z /$ $(2 w)$ the spot size at the focus is $w_{0}=2 \lambda f / \pi$. But. the intensity at the focus of a Gaussian beam is related to the power by

$$
I(0)=2 P /\left(\pi w_{0}^{2}\right) .
$$

Therefore, the energy density $u(0)=I(0) / c$ for a spherical mirror is given by

$$
u(0)=2 P / \pi c w_{0}^{2}=\pi P / 2 c \lambda^{2} f^{2} .
$$

Now we consider the wrap-around focusing element of Fig. 1(b). The device that we have constructed is shown schematically in Fig. 2. A light beam incident from the left is deflected upward, diverged by the conical mirror, and condensed to a focus by the wrap-around mirror. An arrangement of this type provides efficient coupling of a conventional laser beam to a radial propagating beam, but other configurations are also possible. A relationship between the energy density and the input power can again be obtained. The energy distribution is closely related to the modes of radial-mode lasers that have been developed for use as compact laser oscillators and amplifiers. ${ }^{8}$ The asymptotic form for the energy density of the fundamental inward propagating mode is ${ }^{9}$

$$
u(r, z)=[u(0) / \pi k r]\left(w_{0} / w\right) \exp \left(-2 z^{2} / w^{2}\right),
$$

where $u(0)$ is again the energy density at the focus and $w$ is the spot size in the $z$ direction of the diskshaped mode, which is governed by an equation similar in form to Eq. (10). Thus the intensity of the inward traveling beam is

$$
I(r, z)=\frac{c u(0)}{\pi k r}\left(w_{0} / w\right) \exp \left(-2 z^{2} / w^{2}\right) .
$$

The total input power is obtained by integrating the

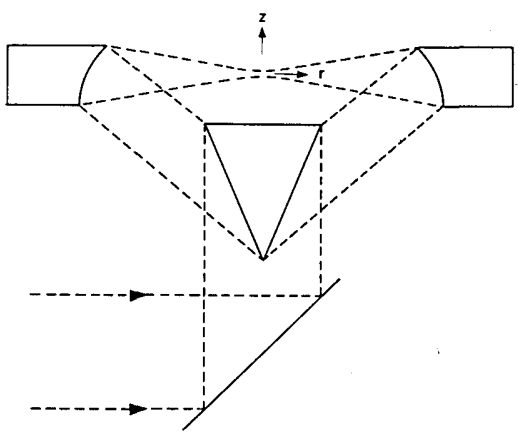

Fig. 2. Schematic drawing of experimental focusing device. 


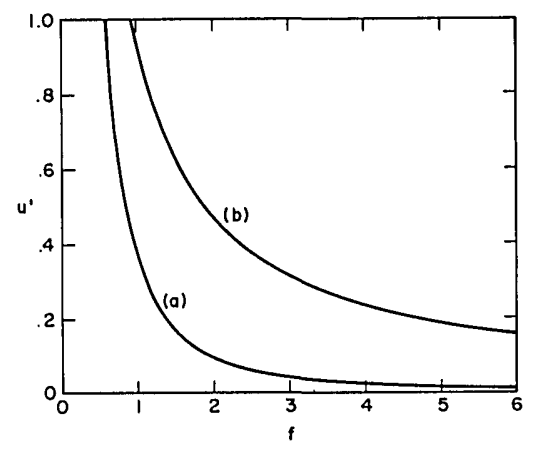

Fig. 3. Normalized energy density at the focus as a function of $f$ number based on Eq. (16) for (a), a spherical mirror and (b), a radial focusing mirror. The $f$ number is not well-defined for $f \lesssim 1$.

intensity from Eq. (13) over the surface of a cylinder of radius $r$ according to

$$
P=\int_{0}^{2 \pi} \int_{-\infty}^{\infty} I(r, z) r d z d \phi=\frac{c \lambda w_{0}}{(2 \pi)^{1 / 2}} u(0) .
$$

In terms of the $f$ number $f=r /(2 w)$ the spot size at the focus is again $w_{0}=2 \lambda f / \pi$. Thus Eq. (14) may be written

$$
u(0)=\pi^{3 / 2} P / 2^{1 / 2} c \lambda^{2} f .
$$

For comparison the previous results may be collected together. If the energy density at the focus is normalized in terms of the input power using the parameter $u^{\prime}=3 c \lambda^{2} u(0) /(4 \pi P)$, then Eqs. (9), (11), and (15) may be written

$$
u^{\prime}= \begin{cases}1 & \text { paraboloidal } \\ 3 / 8 f^{2} & \text { spherical } \\ 3 \pi^{1 / 2} / 2^{5 / 2} f & \text { radial mode }\end{cases}
$$

Equations (16) are plotted in Fig. 3. Evidently for reasonable $f$ numbers, the radial focusing device is much better than a conventional spherical mirror in terms of its energy collection properties. When $f=$ 4 , for example, the spherical mirror is poorer by a factor of 10. Also, since the energy at the focus of the spherical mirror is in the form of a traveling wave, only half the energy on the average is contained in the electric field. With the radial-mode mirror, on the other hand, the fields at the focus are standing waves, so that all of the energy may be contained in either the electric or magnetic fields, depending on the choice of modes. This additional factor of 2 could be important in applications such as gas breakdown, where it is basically the electric-fielddriven cascade ionization that initiates the discharge.

For the interaction with objects that are large compared to a wavelength, the maximum energy density per watt becomes a less important consideration than the symmetry of the illumination. Still the infinitely deep paraboloidal mirror represents the ideal. In the geometrical optics limit an input beam that is properly matched to the paraboloidal mirror yields spherically symmetric illumination. A straightforward calculation shows that the incident beam should have an intensity distribution governed by

$$
I(r)=p^{2} P / \pi\left(p^{2}+r^{2}\right)^{2},
$$

where $p$ is the radius of curvature of the paraboloid at its vertex and $P$ is the total incident power. This ideal situation can be well approximated by a real paraboloidal mirror and an incident Gaussian beam with the intensity profile $I(r)=2 P /\left(\pi w^{2}\right)$ exp$\left(-2 r^{2} / w^{2}\right)$. The two intensity distributions are equal at their half-intensity points (the input beam will approximately match the mirror) if the spot size of the incident beam is related to the parameter $p$ of the mirror by $w=\left[2\left(2^{1 / 2}-1\right) / \ln 2\right]^{1 / 2} p \simeq 1.1 p$. In the radial focusing device the focal region is illuminated from an angle of $360^{\circ}$. A conventional spherical mirror, on the other hand, can illuminate a target from only one side. These optical symmetry considerations are important for applications involving optical pumping, heating, or compression. The hope of obtaining pellet compression for laser fusion, for example, currently rests on approximating spherical symmetry with a large number of conventional beams.

Another aspect is ease of fabrication. We find that a radial-mode mirror is basically no more difficult to construct than a conventional spherical mirror. The device described in the next section was shaped and polished simply on a lathe. By contrast the deep paraboloidal mirror contour is much more difficult to develop. Finally, accessibility of the focal spot is also important. The focus in a paraboloidal mirror is obscured by the mirror itself. With a simple spherical mirror the focus is visible, but it cannot be approached by experimental apparatus without obstructing the input beam (a tilted ellipsoidal mirror overcomes this difficulty). By contrast the focus of the radial-mode element is readily accessible for insertion of samples for illumination or instrumentation for measurements.

\section{Experiment}

The radial focusing device is shown schematically in Fig. 2. The mirrors are fabricated from aluminum using simple machining and polishing techniques. The inner diameter of the wrap-around mirror is about $22 \mathrm{~cm}$, and the curvature is chosen to yield a focus at the center. This sharp focusing in the $z$ direction is essential for gas breakdown but it might not be desirable for some optical pumping applications. The conical mirror is supported by a thin metal strip, which has a negligible effect on the beam. Precise positioning adjustments are included for the plane and conical mirrors so that the alignment sensitivity can be investigated. The laser used in these experiments is a low-mode-order (plane parallel) double-discharge $\mathrm{CO}_{2}$ TEA instrument with a power capability of up to $100 \mathrm{MW}$ at $10.6 \mu$ wavelength.

In Fig. 4 is a top view of an air breakdown spark in the focusing device. The dimensions of the spark are 


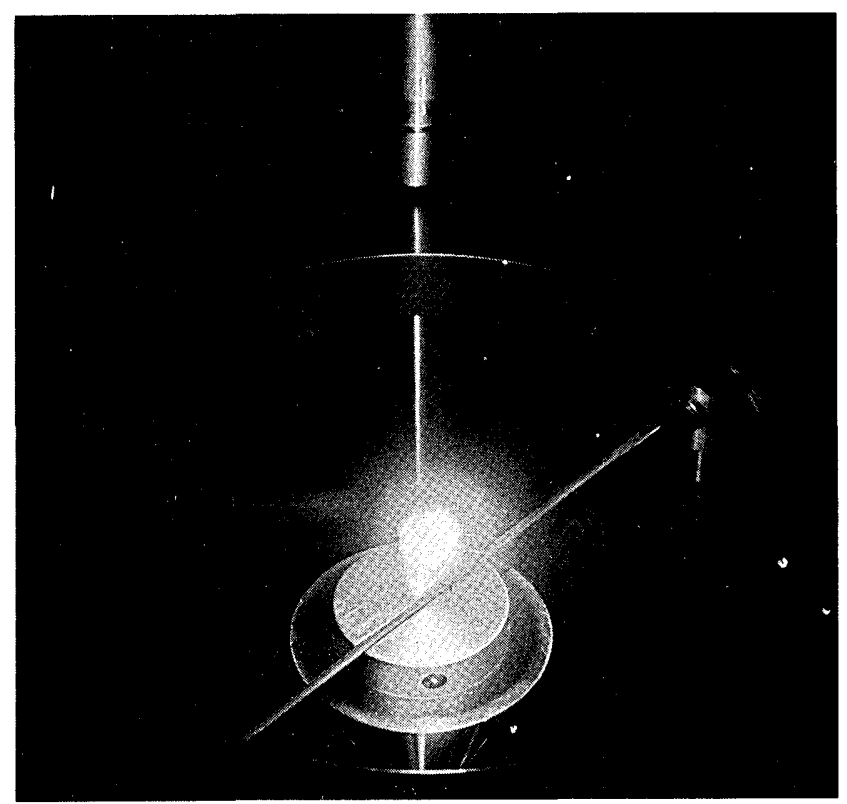

Fig. 4. Air breakdown spark in the radial focusing mirror.

determined by the geometry of the laser beam near the focus. In appearance the breakdown is a thin disk-shaped plasma about $1.5 \mathrm{~cm}$ in diameter, and the behavior of this spark yields a direct visual indication of the focusing properties and alignment sensitivity of the mirror. It is found, for example, that the spark retains its disk appearance if the conical mirror is tilted by as much as about $0.5^{\circ}$. However, for larger mirror tilts there is a separation into two distinct sparks. The positions of these sparks follow simply from ray-tracing arguments and the change in effective focal length of the wrap-around mirror as the beam direction changes.

Laser breakdown of a gas typically proceeds in the following fashion. The discharge is initiated due to the presence of an impurity or background ionization in the focal region. The discharge grows by a process of cascade ionization, becomes opaque to the laser radiation, and expands toward the laser beam as a laser-driven shock. ${ }^{1}$ With the present focusing device the shock propagates radially outward until it becomes transparent to the laser beam. Left behind is a more-or-less uniformly glowing plasma sustained by the nitrogen-pumped tail of the $\mathrm{CO}_{2}$ TEA laser pulse.

In Fig. 5(a) is a plot of the maximum discharge radius $r_{m}$ as a function of the peak laser power $P$. The radius measurements were made by imaging the focal region onto a screen, and the power measurements were made with a photon drag detector. The sparks expand until the intensity at the leading surface is too small to sustain the plasma. But at distances from the focus that are much greater than the Rayleigh length

$$
r_{0}=\pi w_{0}^{2} / \lambda \simeq 0.2 \mathrm{~mm}
$$

the surface area of the advancing shock is propor- tional to $r^{2}$, so it is expected that the maximum radius of the spark should be roughly proportional to the square root of the incident power. This squareroot dependence is fitted to the data in Fig. 5(a). From the known spark dimensions and the incident beam power it is found that the intensity necessary to sustain the shock is about $10^{8} \mathrm{~W} / \mathrm{cm}^{2}$.

The time evolution of the discharge is studied by means of photomultipliers at the image of the spark. One photomultiplier is placed at the center of the image and another is placed nearer the edge. The time difference between the initial photomultiplier responses together with the magnification of the imaging system yields the radial velocity of the laser-driven shock. These measurements indicate that the shock wave propagates outward at a velocity of about $6 \times 10^{6} \mathrm{~cm} / \mathrm{sec}$, which is comparable to streak camera results with conventional breakdown sparks. ${ }^{10}$

In Fig. 5(b) is shown a typical time display of the light output from a breakdown spark. For this example a photodiode was positioned at the image of a point $2 \mathrm{~mm}$ from the focus. The breakdown pulse and afterglow are clearly shown. Closer to the focus the initial pulse is more dominant, while at large distances the pulse is weak compared to the laserpumped glow. The entire discharge is always surrounded by a much larger faint spherical luminous region that is ionized by radiation from the spark. ${ }^{11}$ At a power level of $60 \mathrm{MW}$ this region is about $5 \mathrm{~cm}$ in diameter.

\section{Conclusion}

The theoretical energy-focusing properties of several basic mirror configurations have been derived and compared. It has been shown that a new radial focusing device has the useful features of high energy density, symmetric illumination, and straightforward construction. These results have been verified ex-
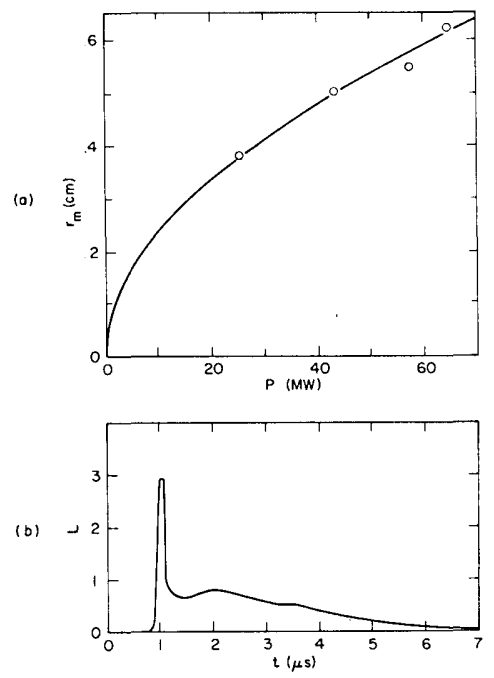

Fig. 5. Shown here are $(a)$ the spark radius $r_{m}$ as a function of the laser power $P$ and $(b)$ the light output from the spark $L$ in arbitrary units vs time from the laser discharge. 
perimentally by investigations concerning the breakdown spark that results when a high powered laser beam is tightly focused in air. The geometry, time evolution, and alignment sensitivity of these sparks are in agreement with the theoretical considerations. While only pulsed sparks have been obtained, the improved energy concentration properties should be especially useful for cw breakdown, since conventional cw lasers have barely adequate output power for breakdown, even with pressurized gases. Besides the generation of extremely clean electrodeless plasmas, other specific applications include optical pumping of lasers oriented along the $z$ axis of the focusing device, zone refining of cylindrical samples, and possibly investigations involving the compression and fusion of DT pellets.

The authors wish to acknowledge valuable discussions with M. E. Marhic. This work was supported in part by the National Science Foundation.

\section{References}

1. C. DeMichelis, IEEE J. Quantum Electron. QE-5, 188 (1969) and references.

2. N. A. Generalov, V. P. Zimakov, G. I. Kozlov, V. A. Masyukov, and Yu. P. Raizer, Sov. Phys. JETP 34, 763 (1972).

3. D. L. Franzen, Appl. Phys. Lett. 21, 62 (1972).

4. J. Longfellow, in Lasers in Industry, S. S. Charschan, Ed. (Van Nostrand Reinhold Co., New York, 1972), p. 305.

5. J. S. Clarke, H. N. Fisher, and R. J. Mason, Phys. Rev. Lett. 30,89 (1973).

6. S. Ramo, J. R. Whinnery and T. Van Duzer, Fields and Waves in Communication Electronics (Wiley, New York, 1965), p. 556.

7. H. Kogelnik and T. Li, Appl. Opt. 5, 1550 (1966).

8. L. W. Casperson, J. Opt. Soc, Am. 63, 25 (1973).

9. L. W. Casperson and C. Romero, IEEE J. Quantum Electron. QE-9, 484 (1973).

10. M. Gravel, W. J. Robertson, A. J. Alcock, K. Büchl, and M. C. Richardson, Appl. Phys. Lett. 18, 75 (1971).

11. G. A. Askaryan, M. S. Rabinovich, M. M. Savchenko, and A. D. Smirnova, JETP Lett. 1, 162 (1965).

w.C. Schwartz, President of International Laser Systems, Inc. of Orlando, Florida announced that the assets of optical Communications, Inc., also of Orlando have been acquired from Andersen Laboratories, Inc.

The new operation, to be known as the OpCom Division, will be the base for further expansion into commercial laser products. Products produced by OpCom include laser data communication systems, a laser construction alignment system and a laser microwelder.

Warren Birge, previously President of Optical Communications, has been appointed Director of the new division. 